\title{
Estudio de la capacidad productora de biofilm en Streptococcus equi subsp. equi
}

\author{
Bustos, C.P.; Marfil, M.J.; Lanza, N.S.; Guida, N. \\ Universidad de Buenos Aires, Facultad de Ciencias Veterinarias, Cátedra de Enfermedades Infecciosas, \\ Buenos Aires, Argentina. E-mail: carlabustos@fvet.uba.ar
}

\begin{abstract}
Resumen
Bustos, C.P.; Marfil, M.J.; Lanza, N.S.; Guida, N.: Estudio de la capacidad productora de biofilm en Streptococcus equi subsp. equi. Rev. Vet. 28: 1, 3-8, 2017. Luego de adherirse, las bacterias pueden desarrollarse en forma de biofilm, es decir, una comunidad de células bacterianas adheridas y envueltas en una matriz extracelular producida por ellas mismas. Streptococcus equi subsp. equi (S. equi) es una bacteria adaptada al equino que produce la adenitis equina y que puede permanecer en nasofaringe y bolsas guturales de animales recuperados que actúan como portadores y fuentes de infección. Diversos investigadores han propuesto la producción de biofilm bacteriano como modelo de infecciones crónicas y de portadores asintomáticos en seres humanos y animales. Por lo tanto, el objetivo de este trabajo fue evaluar la capacidad productora de biofilm de S. equi. Se trabajó con 84 aislamientos de $S$. equi utilizando el método colorimétrico de la microplaca y el del portaobjeto, observando microscópicamente la presencia de polisacárido extracelular (PSE) y la formación de aglomerados celulares. En el método colorimétrico, se obtuvieron valores homogéneos a las $18 \mathrm{~h}$ de incubación, sin encontrarse diferencias con los distintos medios testeados $(\mathrm{p}>0,05)$. La mayor producción fue a las $36 \mathrm{~h}$ de incubación, alcanzándose los valores más altos en presencia de plasma equino $(\mathrm{p}<0,05)$. El 66\% de los aislamientos presentó PSE, sin observarse diferencias entre los aislamientos de enfermos y de portadores. Se encontró asociación entre la formación de aglomerados celulares y producción de PSE $(\mathrm{p}<0,05)$. Se logró demostrar por primera vez la capacidad de $S$. equi para producir biofilm, observándose la formación de una sustancia compatible con el PSE que podría favorecer la permanencia de la bacteria en los caballos portadores. Además, algún componente plasmático del equino sería estimulante de la formación de biofilm como se ha observado en Streptococcus zooepidemicus. Se propone profundizar en el estudio del biofilm para contribuir al conocimiento del comportamiento de S. equi en los portadores.
\end{abstract}

Palabras clave: equino, Streptococcus equi subsp. equi, biofilm, resistencia bacteriana.

\begin{abstract}
Bustos, C.P.; Marfil, M.J.; Lanza, N.S.; Guida, N.: Study of the biofilm production capacity in Streptococcus equi subsp. equi. Rev. Vet. 28: 1, 3-8, 2017. After attached, bacteria can grow in biofilms as a community of cells surrounded by an extracellular matrix synthesized by themselves. Streptococcus equi subsp. equi (S. equi) is a microorganism that produces strangles in horses. It can remain in nasopharynx and guttural pouches of recovered animals, situation that plays an important role in the dissemination of the infection. Some researchers have proposed the production of bacterial biofilm as a model of chronical infection and asymptomatic carriers in animals and human beings. The aim of this work was evaluate the capacity of biofilm production of $S$. equi. Eighty-four isolates of $S$. equi were studied by colorimetric and glass slides methods showing the presence of extracellular polysaccharide (PSE) and cell agglomerates. Values were obtained by a colorimetric method at $18 \mathrm{~h}$ of incubation and no difference was found with different culture media $(p>0.05)$. The biggest production of biofilm was obtained at $36 \mathrm{~h}$ of incubation with highest values in the presence of equine plasma $(p<0.05)$. Sixty-six percent of isolates showed PSE and no difference was observed between isolates of carriers and sick animals. There was a significant correlation between agglomerate and PSE formation $(\mathrm{p}<0.05)$. This is the first report of the capacity of biofilm production of $S$. equi and the presence of a substance compatible with extracellular polysaccharide which may favor the persistence of bacteria in carrier horses. Furthermore, some plasmatic component may be facilitating the formation of biofilm as it was previously
\end{abstract}


observed for Streptococcus zooepidemicus. Further assays are needed to study this virulence factor to understand the behavior of $S$. equi in carrier horses.

Key words: equine, Streptococcus equi subsp. equi, biofilm, bacterial resistance.

\section{INTRODUCCIÓN}

Cuando una bacteria alcanza la piel y las mucosas debe poner en juego mecanismos de adherencia para poder colonizar. Estos mecanismos pueden ser inespecíficos de tipo físico-químico o específicos en los que intervienen estructuras denominadas adhesinas. Luego de adherirse, las bacterias pueden desarrollarse en forma de biofilm permaneciendo adheridas a una superficie ya sea biótica o abiótica. Existen muchas definiciones de biofilm, pero todas concuerdan en que se trata de una comunidad de células bacterianas que crecen envueltas en una matriz extracelular producida por ellas mismas y que está adherida a una superficie ${ }^{15,18}$.

La composición de la matriz extracelular o slime dependerá del microorganismo, pero en general tiene como componente principal al agua y diferentes proporciones de otras sustancias tales como polisacáridos, proteínas, ADN, lípidos, glicolípidos y diversos iones 9, 11, 14, 25 . Resulta interesante que las bacterias pueden vivir como células planctónicas o flotantes con metabolismo activo capaces de proliferar y propagarse, o como células sésiles o unidas para persistir ${ }^{4,8,18}$.

Streptococcus equi subsp. equi (S. equi) es una bacteria adaptada al equino que produce la adenitis equina, una enfermedad infecciosa que cursa con faringitis, rinitis y linfoadenitis de los linfonódulos de cabeza y cuello de caballos jóvenes 1, 5, 23, 24, 26 .

La enfermedad es mundialmente distribuida y afecta a la producción hípica de nuestro país ocasionando múltiples brotes que provocan pérdidas económicas. Luego de superado el cuadro clínico de la enfermedad, los animales pueden continuar portando al agente en la nasofaringe y bolsas guturales por largos periodos de tiempo, actuando como fuente de infección para otros animales susceptibles $1,5,16,17,23$.

Recientemente fue descripta la formación de biofilm en estreptococos relacionados con $S$. equi; en Streptococcus equi subsp. zooepidemicus (S. zooepidemicus) se ha demostrado la expresión de proteínas en el biofilm asociadas al metabolismo, la adhesión y condiciones de estrés ${ }^{28}$ y en Streptococcus disgalactiae subsp. equisimilis se obtuvieron resultados que sugieren que el biofilm sería inmunogénico y de una composición que incluye proteínas, extrapolisacáridos y ADN extracelular ${ }^{10}$.

Muchas infecciones pueden volverse crónicas y las bacterias permanecer en los tejidos del huésped sin signología clínica en el caso de los portadores, o con signología leve en las infecciones subclínicas. Se ha propuesto por diversos investigadores la producción de biofilm bacteriano como modelo de infecciones crónicas en seres humanos y animales $3,4,6,8,9,11,15$. Un aspecto relevante a tener en cuenta sería el papel que desempeñan las biopelículas como formas de resistencia ante agentes antimicrobianos, desinfectantes y germicidas ${ }^{9,20}$.

Para ejercer su acción, el antibiótico tiene que ser capaz de penetrar en el biofilm, lo cual depende de la estructura del mismo, así como de las características bioquímicas de la matriz que se establece alrededor de la biopelícula. Se ha observado que el establecimiento de biopelículas de Streptococcus pneumoniae (S. pneumoniae) sobre la superficie de las mucosas favorece la supervivencia de la bacteria y permanencia en tracto respiratorio de personas ${ }^{6,15} \mathrm{y}$ se ha comparado la susceptibilidad antibiótica bacteriana en formaciones de biofilm y en células planctónicas, observándose que estas últimas mostraban mayor sensibilidad a un número más elevado de antibióticos ${ }^{19}$.

El proceso de formación de biofilm es complejo y varía entre microorganismos, pero en todos ellos podemos distinguir las mismas etapas. El primer paso para que la biopelícula se produzca es la adherencia bacteriana a una superficie que en principio es lábil y posteriormente se vuelve irreversible ${ }^{3,11,14,25}$. Luego, comienza la proliferación bacteriana y la producción y excreción de los componentes de la matriz extracelular formándose multicapas celulares embebidas en este slime ${ }^{11,25}$.

En la etapa de maduración se produce un biofilm maduro y resistente, con un complejo sistema de comunicación en el que las bacterias se comunican y expresan o reprimen genes a través del quorum sensing ("autoinducción") 9, 12, 13,21. La última etapa -denominada dispersión- se caracteriza por la producción de enzimas que lisan el slime, y la diferenciación de un subgrupo de bacterias que pueden cambiar su fenotipo a planctónicas nuevamente y dispersarse para adherirse a otros sustratos y comenzar otra vez el proceso $9,11,25$.

Siendo el biofilm un potencial factor de patogenicidad que puede favorecer la permanencia de microorganismos en los tejidos del huésped, el objetivo de este trabajo fue evaluar la capacidad productora de biofilm de $S$. equi como favorecedora de la persistencia del agente en caballos portadores de adenitis equina.

\section{MATERIAL Y MÉTODOS}

Aislamientos. Se utilizaron aislamientos de S. equi obtenidos a partir de equinos con signos clínicos de adenitis equina y de caballos clínicamente sanos recuperados de la enfermedad. Las muestras fueron procesadas en el laboratorio de la Cátedra de Enfermedades Infecciosas de la Facultad de Ciencias Veterinarias de la Universidad de Buenos Aires. 
Método colorimétrico en microplaca. Se sembraron los aislamientos en el medio Todd Hewitt Broth (THB) suplementado con $0,2 \%$ de extracto de levadura (EL) y $10 \%$ de suero de equino adulto (SEA) y se incubaron en atmósfera enriquecida con $\mathrm{CO}_{2}$ over nigth. Se diluyó cada cultivo 1/10 en medio fresco de THB suplementado con $0,2 \%$ de EL y $10 \%$ de SEA con y sin agregado de glucosa filtrada al $0,1 \%$ y en medio fresco de THB suplementado con $0,2 \%$ de EL y $10 \%$ de plasma equino. Se colocaron $200 \mu 1$ de cada dilución en cada pocillo de una microplaca de 96 pocillos de fondo plano. Luego de incubar por 18 y $36 \mathrm{~h}$, los pocillos se lavaron 2 veces con agua destilada estéril y se colorearon con violeta de genciana al $1 \%$ durante 10 min. Luego de lavar 2 veces con agua destilada estéril, se eluyeron con $200 \mu \mathrm{l}$ de etanol $95 \%$. Se determinó la densidad óptica (DO) a $570 \mathrm{~nm}$ para cada aislamiento y para cada blanco (medio estéril) en el lector de ELISA (Dynex, MRX Revelation). Se determinó la DO corregida de cada aislamiento para las 18 y $36 \mathrm{~h}$ de incubación con la siguiente fórmula: DO 570 aislamiento corregida $=$ DO 570 aislamiento - DO 570 blanco.

Método del portaobjeto. El protocolo de trabajo se basó en las técnicas utilizadas por otros investigadores ${ }^{3,18}$ con modificaciones. Se incubaron los aislamientos en THB suplementado con $0,2 \%$ de EL y $10 \%$ de SEA y se incubaron en atmósfera enriquecida con $\mathrm{CO}_{2}$ over nigth. Luego de diluir cada cultivo 1/10 en medio fresco, se colocó $1 \mathrm{ml}$ de esa dilución sobre un portaobjeto nuevo y estéril. Cada uno se incubó dentro de una placa de Petri estéril a $37^{\circ} \mathrm{C}$ en atmósfera enriquecida con $\mathrm{CO}_{2}$ durante $18 \mathrm{~h}$. Se procedió a lavar 3 veces con agua destilada estéril y fijar durante $1 \mathrm{~min}$ con metanol. Se tiñeron con alcian blue $4 \mathrm{X}$ al $2 \%$ durante $10 \mathrm{~min}$. Posteriormente, se coloreó con violeta de genciana al $4 \%$ durante $30 \mathrm{seg}$. Se observaron en microscopio óptico (Leica DM500, Alemania) a 10, 40 y 100 aumentos. Se observó la adherencia bacteriana, la agrupación y la presencia de un potencial polisacárido extracelular (PSE). Las imágenes se capturaron con microscopio de campo claro trilocular Leica DM4000B LED y cámara Leica DCC-380X con soporte digital para captura de imágenes LASZ Leica Inc.

Análisis estadístico. Para estudiar el nivel de asociación entre las variables cualitativas se realizó la prueba de chi cuadrado con un nivel de significancia de $\mathrm{p}<0,05^{22}$. Para el análisis de las variables cuantitativas se realizó la prueba de Wilcoxon con un nivel de significancia de $\mathrm{p}<0,05^{22}$. Para todas las pruebas estadísticas se trabajó con el software InfoStat versión $2015^{7}$.

\section{RESULTADOS}

Método colorimétrico en microplaca. Se trabajó con 81 aislamientos, 66 obtenidos de caballos enfermos y 15 provenientes de equinos portadores. Se determinó la DO 570 corregida de cada aislamiento para los diferentes medios y tiempos de incubación. En el gráfico de bastones (Figura 1) se observa homogeneidad en los

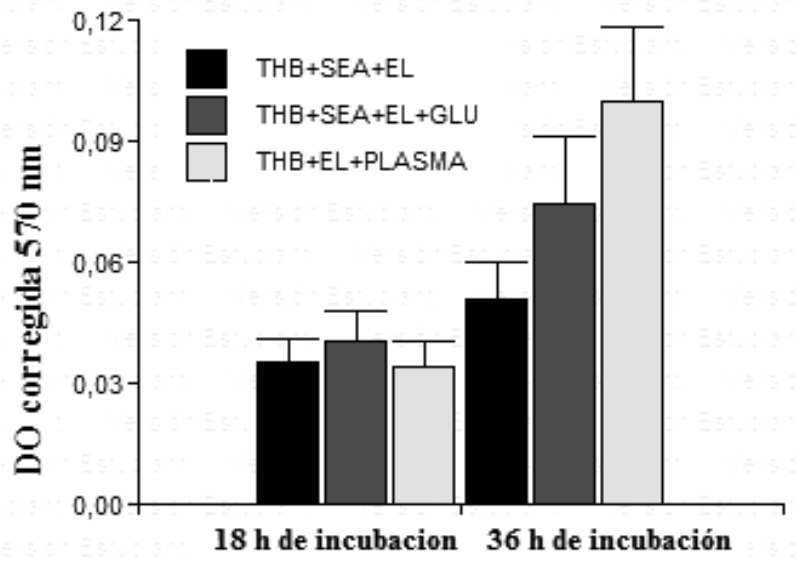

Figura 1. Resultados del método de biofilm en microplaca en los diferentes medios y tiempos de incubación, para el total de los aislamientos. THB: Todd Hewitt Broth; SEA: suero de equino adulto; EL: extracto de levadura; GLU: glucosa; PLASMA: plasma de equino; DO: densidad óptica.

valores de DO alcanzados en los tres medios testeados a las $18 \mathrm{~h}$ de incubación, y valores heterogéneos a las $36 \mathrm{~h}$ de incubación. Además, puede observarse que los valores de DO a las $36 \mathrm{~h}$ de incubación son mayores que a las $18 \mathrm{~h}$, alcanzándose los valores más altos en la incubación en presencia de plasma equino.

Método del portaobjeto. Se trabajó con 89 aislamientos, 73 obtenidos de equinos enfermos y 16 a partir de caballos portadores. Se registró la agrupación de los cocos clasificándose en cocos en cadenas y en aglomerados pequeños y aglomerados grandes (Figura 2) según el tamaño de los mismos (punto de corte 100 $\mu \mathrm{m})$. El 33\% de los aislamientos se agrupó en cocos en cadenas, el $27 \%$ en aglomerados pequeños y el $40 \%$ en aglomerados grandes. Al comparar los aislamientos obtenidos de enfermos con los de portadores, surge que el $32 \%$ y el $38 \%$ se agruparon como cocos en cadena, el $29 \%$ y $19 \%$ como aglomerados pequeños y $39 \%$ y $44 \%$ como aglomerados grandes, respectivamente. Con respecto a la presencia de un potencial PSE, se clasificó como presente o ausente. Se consideró al PSE presente cuando se observaba una sustancia coloreada con el alcian blue alrededor de los cocos y PSE ausente cuando no se observaba la coloración celeste o sólo se observaba alrededor de los cocos aislados ya que esta última sustancia correspondería a la cápsula y no al PSE (Figura 2). El 66\% de los aislamientos presentó PSE. Si comparamos los grupos de aislamientos, el $65 \%$ de los aislamientos de enfermos y el 69\% de los aislamientos de portadores, presentaron PSE. Por otro lado, en el grupo de aislamientos productores de PSE, el 96\% de ellos se agrupó en aglomerados, mientras que en el grupo de aislamientos no productores de PSE sólo el 10\% de ellos se agrupó en aglomerados más pequeños.

Análisis estadístico. No se encontraron diferencias significativas en la producción de biofilm en microplaca a las $18 \mathrm{~h}$ de incubación en los diferentes medios $(p>0,05)$. Se encontraron diferencias significativas en- 

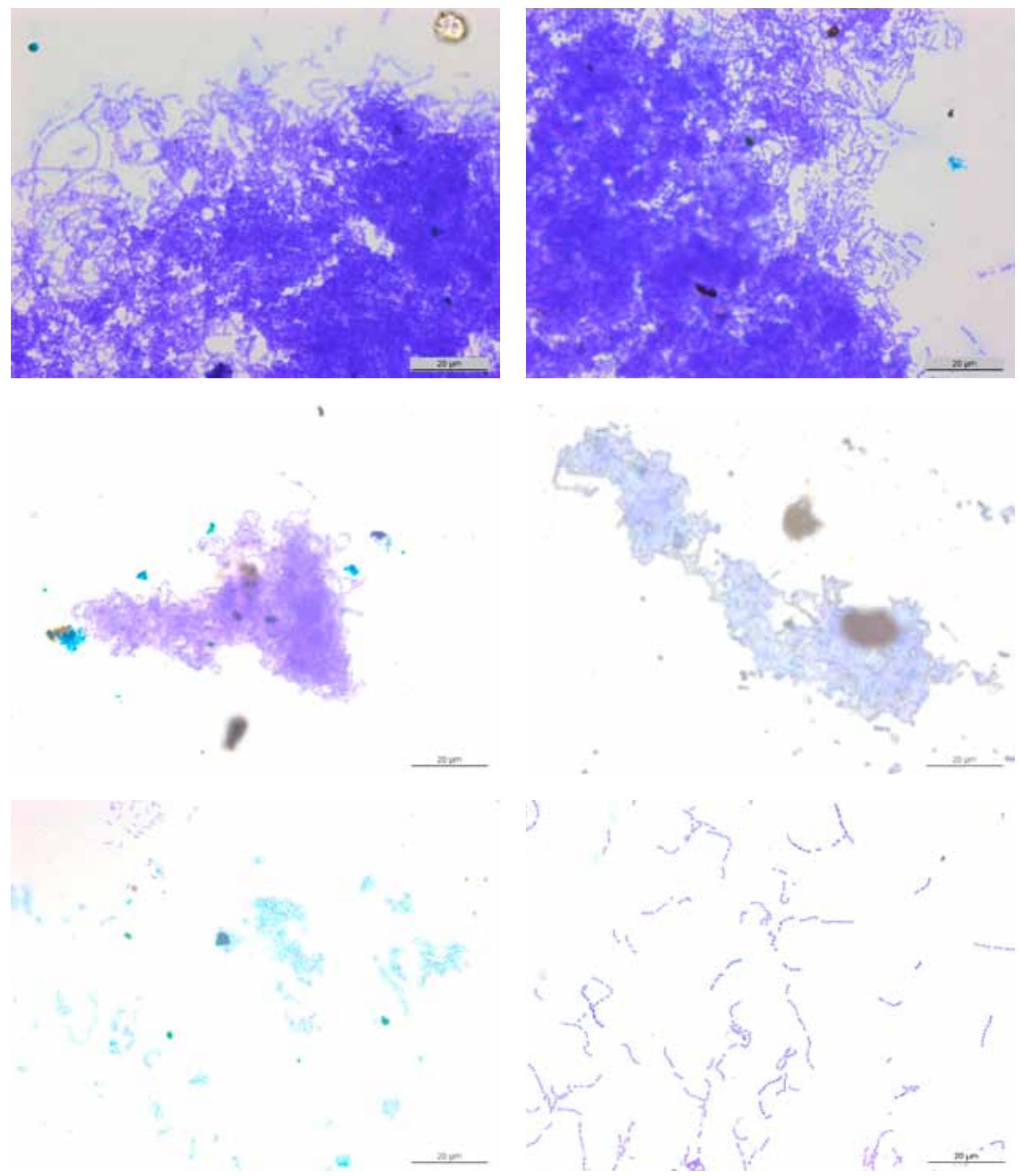

Figura 2. Formación de biofilm en portaobjetos mostrando aglomerados grandes $(A-B)$ y pequeños $(C-D-E)$ con diferentes densidades bacterianas y cantidad de polisacárido extracelular (PSE), así como cocos en cadenas aislados (F) sin PSE (100 aumentos).

tre la producción con SEA y plasma equino $(\mathrm{p}=0,0068)$, entre SEA y glucosa $(\mathrm{p}=0,0310)$ y también entre plasma equino y glucosa $(\mathrm{p}=0,0111)$ a las $36 \mathrm{~h}$ de incubación. Al comparar la producción de biofilm en microplaca en el mismo medio a las 18 y $36 \mathrm{~h}$ de incubación, no se encontraron diferencias estadísticamente significativas en la producción de biofilm con SEA $(p=0,1620)$ pero si se encontraron en la producción de biofilm con glu$\operatorname{cosa}(\mathrm{p}=0,0311)$ y de plasma equino $(\mathrm{p}=0,218)$. Para la formación de biofilm en portaobjetos, se encontró aso- ciación estadística entre la agrupación en el biofilm y la formación de PSE $(p<0,0001)$.

\section{DISCUSIÓN}

Se logró demostrar la capacidad de $S$. equi para producir biofilm mediante los métodos colorimétrico en microplaca y portaobjetos. Este hallazgo es destacable ya que no se encontró bibliografía que mencione la detección de este factor de patogenicidad en dicho microorganismo. 
Se pudo trabajar con el método de la microplaca, que es el más difundido para el estudio inicial del biofilm microbiano y tiene la ventaja de ser una técnica cuantitativa y relativamente fácil de realizar, permitiendo el estudio en diferentes condiciones de incubación. Este método fue utilizado por varios investigadores para el estudio de biofilm en otros estreptococos y se basa en la capacidad de adherencia bacteriana al poliestireno ${ }^{3,10,14,15,18,27}$.

Es importante resaltar que complementar esta técnica con la detección de un potencial PSE presente en las agrupaciones celulares de estreptococos, resulta de utilidad para diferenciar la adherencia de la formación de biofilm ${ }^{3,18}$. Por otro lado, hay que considerar que la cinética de formación del biofilm varía entre microorganismos por lo que es necesario testear diferentes condiciones de incubación.

En nuestro estudio se observó mayor cantidad de aislamientos produciendo biofilm temprano que tardío. Sin embargo, el nivel de producción de biofilm tardío fue mayor, alcanzando valores de DO corregidos mayores a las $36 \mathrm{~h}$ de incubación. También se constató que, a las $18 \mathrm{~h}$, la producción de biofilm fue homogénea mientras que a las $36 \mathrm{~h}$ se observó una mayor variación en la producción de biofilm, lo cual se confirmó estadísticamente. Esto podría evidenciar que el desarrollo de biofilm temprano sería independiente de las características del medio, pero que para la formación de biofilm tardío, las características del medio serían influyentes.

Si se compara la producción de biofilm en un mismo medio en los diferentes tiempos de incubación, se observan niveles mayores de producción de biofilm al incubarse en presencia de plasma, siendo mayor esa diferencia a las $36 \mathrm{~h}$ de incubación.

Actualmente, se sabe que la mayoría de las bacterias se encuentran formando biofilm, aunque un ambiente hostil provocaría el cambio de células planctónicas a células en biofilm 9, 11, 14,25. Sin embargo, los resultados obtenidos sugieren que en $S$. equi la presencia de plasma equino y de glucosa serían necesarios para el proceso de producción de biofilm, quizá por ser nutrientes requeridos para la génesis de la matriz extracelular o por participar en la señalización entre células, como sucede en otros géneros bacterianos y fue tratado en la sección introducción.

Estudios recientes, reportan la formación in vitro de biofilm de $S$. zooepidemicus incubándolo en presencia de fibrinógeno durante $24 \mathrm{~h}$, obteniendo muy buenos resultados, que fueron confirmados mediante estudios proteómicos ${ }^{27,28}$. Quizá algún componente plasmático del equino sea relevante en las infecciones crónicas asociadas al biofilm en el caso de $S$. zooepidemicus y en la persistencia de $S$. equi en los portadores de adenitis equina.

Como ya se mencionó, con la coloración alcian blue, que tiñe sustancias de naturaleza polisacárida, se puede identificar una sustancia que rodea a las bacterias, compatible con el PSE comúnmente encontrado en el biofilm. Como en otros estudios ${ }^{3,18}$, se pudo observar la disposición de las bacterias en estructuras similares a microcolonias y una sustancia compatible con PSE entre los microorganismos (Figura 2). Se trabajó con 18 $\mathrm{h}$ de incubación porque se quería evaluar la presencia de PSE en la mayoría de los aislamientos, y al poner a punto el protocolo de trabajo, al igual que lo ocurrido en el método de microplaca, no se observaron diferencias con los distintos medios de cultivo testeados.

Si se comparan los aislamientos de enfermos y los de portadores, los porcentajes fueron similares, pero hay que considerar que el número de aislamientos de portadores fue menor y que se deberían realizar más estudios aumentando la cantidad de aislamientos. Se encontró asociación estadística entre la formación de aglomerados y la producción de PSE, encontrándose que casi todos los aislamientos productores de PSE se agruparon en aglomerados.

Estos resultados serían esperables ya que la matriz extracelular actuaría como una sustancia que adhiere a las bacterias al soporte y entre ellas mismas con la formación de aglomerados, siendo su función la de proteger a los microorganismos que la producen de agentes externos, sustancias antibacterianas y defensas del huésped.

Se ha observado que el crecimiento en biofilm de S. pneumoniae sobre la superficie de las mucosas favorece la supervivencia de la bacteria y su consecuente permanencia en el tracto nasofaringeo y las vías aéreas inferiores en seres humanos ${ }^{6,15}$. Al igual que en $S$. pneumoniae, la formación de biofilm por $S$. equi podría favorecer la permanencia de la bacteria en nasofaringe y bolsas guturales en los caballos portadores de adenitis equina, por aumentar la adherencia de la bacteria al epitelio y por la acción protectora de la matriz extracelular.

Hay que considerar que ciertas infecciones asociadas con biofilms pueden llegar a ser hasta 1000 veces más resistentes a las terapias con antimicrobianos ${ }^{2,11,19}$. Esta resistencia antimicrobiana detectada en el biofilm podría relacionarse a la barrera física que produce la matriz extracelular, pero también al estado de quiescencia que poseen las células ${ }^{6,11}$.

En conclusión, este es el primer reporte de la capacidad productora de biofilm de S. equi. Posiblemente la capacidad de formar biofilm detectada pueda ser un aporte para la comprensión de la portación nasofaríngea y gutural en algunos animales recuperados y tal vez su comportamiento pueda ser similar al de otros agentes que permanecen en vías respiratorias de individuos sanos. Profundizar el estudio del biofilm respecto a su composición, estructura, cinética de formación y susceptibilidad antimicrobiana, serían los próximos temas a investigar.

Agradecimientos. A Alejandro Maruri y a la Cátedra de Histología FCV-UBA por su colaboración en la obtención de las imágenes. A los compañeros de la Cátedra de Enfermedades Infecciosas. Trabajo financiado por UBACyT 20020130100307BA. 


\section{REFERENCIAS}

1. Boyle A. 2011. Streptococcus equi subspecies equi infection (strangles) in horses. Comp Cont Educ Vet 33: 1-7.

2. Ceri H, Olson M, Turner R. 2010. Needed, new paradigms in antibiotic development. Exp Opin Pharm 11: 1233-1237.

3. Christensen G, Simpson W, Younger J, Baddour L, Barret F, Melton D, Beachey E. 1985. Adherence of coagulase-negative staphylococci to plastic tissue culture plates: a quantitative model for the adherence of staphylococci to medical devices. J Clin Microbiol 22: 996-1006.

4. Clutterbuck A, Woods E, Knottenbelt D, Clegg P, Cochrane C, Percival S. 2007. Biofilms and their relevance to veterinary medicine. Vet Microbiol 121: 1-17.

5. Cordoni G, Williams A, Durham A, Florio D, Zanoni R, La Ragione R. 2015. Rapid diagnosis of strangles (Streptococcus equi subspecies equi) using PCR. Res Vet Sci 102: 162-166.

6. del Prado G. 2008. Adherencia de Streptococcus pneumoniae a poliestireno, patogenicidad e interferencia de antibióticos e ibuprofeno. Tesis Doctoral, Univ. Complutense de Madrid, España.

7. Di Rienzo J, Casanoves F, Balzarini M, Gonzalez L, Tablada M, Robledo C. 2015. InfoStat versión 2015. Grupo InfoStat, FCA, Universidad Nacional de Córdoba, Argentina.

8. Domenech M, García E, Moscoso M. 2012. Biofilm formation in Streptococcus pneumoniae. Microb Biotech 5: 455-465.

9. Garrett T, Bhakoo M, Zhang Z. 2008. Bacterial adhesion and biofilms on surfaces. Progr Nat Sci 18: 1049-1056.

10. Genteluci G, Silva L, Souza M, Glatthardt T, Mattos M, Ejzemberg R, Alviano C, Figueiredo A, Ferreira B. 2015. Assessment and characterization of biofilm formation among human isolates of Streptococcus dysgalactiae subsp. equisimilis. Int J Med Microbiol 305: 937-947.

11. Jaques M, Aragon V, Tremblay Y. 2010. Biofilm formation in bacterial pathogens of veterinary importance. Anim Health Res Rev 11: 97-121.

12. Marquina D, Sen A. 2010. Sistemas de quorum sensing en bacterias. Reduca (Biología) 3: 39-55.

13. Miller M, Bassler B. 2001. Quorum sensing in bacteria. Ann Rev Microbiol 5: 165-199.

14. Moscoso M, García E, Lopez R. 2006. Biofilm formation by Streptococcus pneumoniae: role of choline, extracellular DNA, and capsular polysaccharide in microbial accretion. J Bacteriol 188: 7785-7795.
15. Moscoso M, García E, López R. 2009. Pneumococcal biofilms. Int Microbiol 12: 77-85.

16. Newton J, Wood J, Chanter N. 1997. Strangles: long term carriage of Streptpcoccus equi in horses. Eq Vet Educ 9: 98-102.

17. Newton J, Wood J, Dunn K, DeBrauwere M, Chanter N. 1997. Naturally occurring persistent and asymptomatic infection of the guttural pouches of horses with Streptococcus equi. Vet Rec 140: 84-90.

18. Oggioni M, Trappetti C, Kadioglu A, Cassone M, Iannelli F, Ricci S, Andrew P, Pozzi G. 2006. Switch from planktonic to sessile life: a major event in pneumococcal pathogenesis. Molec Microbiol 61: 1196-1210.

19. Olson M, Ceri H, Morck D, Buret A, Read R. 2002. Biofilm bacteria: formation and comparative susceptibility to antibiotics. Can J Vet Res 66: 86-92.

20. Rodríguez J, Pascual A. 2008. Actividad de los antimicrobianos en biocapas bacterianas. Enf Infec Microb Clin 26: 107-114.

21. Rojas Badía M. 2011. Quorum sensing en la asociación beneficiosa de las bacterias con las plantas. Rev Colomb Biotecnol 13: 135-143.

22. Steel R, Torrie J. 1988. Bioestadística: principios y procedimientos, 2da. ed., McGraw Hill, Buenos Aires.

23. Sweeney C, Timoney J, Newton J, Hines M. 2005. Streptococcus equi infections in horses: guidelines for treatment, control, and prevention of strangles. $J$ Vet Int Med 19: 123-134.

24. Timoney J. 2004. The pathogenic equine streptococci. Vet Res 35: 397-409.

25. Vila J, Soriano A, Mensa J. 2008. Bases moleculares de la adherencia microbiana sobre los materiales protésicos. Papel de las biocapas en las infecciones asociadas a los materiales protésicos. Enf Infec Microb Clin 26: 48-55.

26. Waller A, Paillot R, Timoney J. 2011. Streptococcus equi: a pathogen restricted to one host. J Med Microbiol 60: 1231-1240.

27. Yi L, Wang Y, Ma Z, Zhang H, Li Y, Zheng J, Yang Y, Fan H, Lu C. 2014. Biofilm formation of Streptococcus equi ssp. zooepidemicus and comparative proteomic analysis of biofilm and planktonic cells. Current Microbiol 69: 227-233.

28. Yi L, Wang Y, Ma Z, Lin HX, Xu B, Grenier D, Fan HJ, Lu CP. 2016. Identification and characterization of a Streptococcus equi ssp. zooepidemicus immunogenic GroEL protein involved in biofilm formation. Vet Res 18: 47-50. 